\title{
EL POLÍGONO HORTÍCOLA EN EL PAÍS VASCO COMO INTERPRETACIÓN DE LAS INICIATIVAS TERRITORIALES INDUSTRIALES
}

\author{
Juan Cruz Alberdi Collantes \\ Departamento de Geografía, Prehistoria y Arqueología \\ Universidad del País Vasco
}

\section{RESUMEN}

La agricultura desarrollada en la proximidad de los espacios urbanos presenta a menudo unas peculiaridades propias. Entre las actividades observadas habitualmente son las distintas modalidades de producción hortícola las más características, que aportan a estos espacios un auténtico carácter de cinturón hortícola. La cercanía de la ciudad, sin embargo, dificulta el acceso al recurso suelo para unas actividades, las agrarias, que no pueden hacer frente a la especulación urbanística. Con el objeto de promocionar estas funciones se ha apostado en el País Vasco por la creación de polígonos rurales de titularidad pública, que surgen como imitación del industrial pero que, frente a éste, adolece de distintos problemas que ponen en entredicho incluso su continuidad. trial.

Palabras clave: País Vasco, agricultura, horticultura, polígono rural, polígono indus-

\begin{abstract}
Farm activity near to the urban area most of times different to the activity that you can find fart to the city. The most important activities in farm urban areas are green productions, sometimes development green belts. The proximity of the city, however, has a negative influence under the value of the ground and the farmers haven't any options to increase their exploitation. In the Basque Country Government has decided development green production areas, continuing the model that has been development in the industrial sector. Farm model, however, haven't than public help as the industrial and, therefore, is continuity is in difficulties.
\end{abstract}

Key word: Basque Country, agriculture, green productions, farm spaces, industrial spaces.

Fecha de recepción: 27 de octubre de 2004. Fecha de aceptación: 14 de abril de 2005 . 
La respuesta de las instituciones públicas a la crisis industrial que sufre el País Vasco desde el primer quinquenio de los años 80 no se hace esperar y ya, para mediados de esta década, distintos programas de reindustrialización comienzan a materializarse. Las medidas iniciadas son muy diversas aunque en términos generales se produce un evolución de la adjudicación de ayudas directas dirigidas a los sectores más castigados por la crisis hacia el apoyo de medidas orientadas a conseguir una reconversión y modernización de un sector económico estratégico en la economía vasca. En esta última línea se engloban distintas iniciativas orientadas a la promoción de suelo para uso industrial y que tienen por objeto ofrecer edificaciones en condiciones ventajosas para el desarrollo de nuevas iniciativas empresariales, polígonos industriales de intervención pública surgidos para reforzar el tejido industrial de la Comunidad.

La crisis económica y las medidas de reconversión también afectan al agro del País Vasco. El modelo ganadero desarrollado en el caserío no es competitivo en un mercado cada vez más capitalizado e internacionalizado en el que prima producir más y más barato. La capacidad de elaboración se concentra en un reducido número de unidades mientras el resto inicia un proceso que lleva a la marginalización de la actividad. Tan sólo aquéllas que escapan de la línea productivista clásica, como los que desarrollan sistemas de venta sin apenas intermediarios, o nuevas producciones reconocidas por su calidad, parecen presentar ciertas perspectivas de progreso partiendo de una situación totalmente minoritaria.

La reconversión agraria, al igual que la industrial, también está siendo profunda, siendo muchas las iniciativas que se han ido desarrollando. Sin embargo, el carácter cerrado del sector, que necesita renovarse en el seno de la familia para asegurar su continuidad, dificulta la incorporación de nuevos agricultores y proyectos, imposibilitados para acceder al disfrute de un recurso básico, el suelo agrario, que en una región urbana como el País Vasco es vendido a precios astronómicos.

Las intervenciones espaciales también se contabilizan entre aquéllas que pretenden desarrollar el agro vasco. En esta línea, retomando la idea del polígono industrial o «industrialdea», se comienzan a crear agroaldeas o polígonos agrícolas mediante la intervención de agentes públicos, dirigidos preferentemente a la potenciación de producciones hortícolas y a permitir el acceso al suelo a jóvenes agricultores. Esta nueva modalidad, sin embargo, tan sólo retoma la idea de los polígonos industriales. Su aceptación, posibilidades administrativas y presupuestarias, disponibilidad de actuación en materia de suelo o el reconocimiento de sus aportaciones nada tienen que ver con aquéllos. El carácter industrial del País Vasco preferencia otro tipo de actuaciones mientras el espacio agrícola, con demasiada frecuencia, no es sino un soporte sobre el que proponer nuevos usos urbano-industriales.

\section{Industrialdea o polígono industrial: estrategia espacial de desarrollo industrial}

La reestructuración productiva de los años 80 sacude a todo el tejido empresarial originando una gran diversidad de respuestas para superar las consecuencias de la crisis. A medida que avanza la década las medidas de promoción industrial van experimentando un cambio sustancial como consecuencia de las alteraciones aparecidas en el sistema productivo durante el periodo de recesión. En general se evoluciona desde una política vertical en la que primaban las subvenciones al capital (incentivos financieros y fiscales) en zonas puntuales del territorio, aquéllas que con mayor intensidad sufrían la pérdida de empleo, hacia una política industrial que prima medidas de corte horizontal de apoyo empresarial (información, asesoramiento), susceptibles de ser absorbidas geográficamente de un modo más difuso y que lleva a referirse a ellas ya en la década de los noventa como políticas de 
promoción industrial o reindustrialización frente al concepto de reestructuración que se tenía en la década anterior ${ }^{1}$.

Una de las líneas de promoción industrial impulsadas es de corte espacial y está dirigida a ofertar suelo industrial por parte de las instituciones públicas. La disponibilidad de suelo es un factor de atracción de empresas industriales y de servicios. El suelo industrial al ser un recurso limitado y un factor de producción necesario es un bien económico que suele estar sometido a procesos de especulación. El problema de déficit de suelo en el País Vasco Atlántico está además condicionado por una orografía en la que predominan las pendientes acusadas y escasean las zonas llanas.

La importancia que las medidas espaciales tienen en la promoción industrial del País Vasco quedan de manifiesto en el carácter estratégico que ha tenido y mantiene en la actualidad el programa Industrialdeak. Esta iniciativa fue fundada en 1982 por el SPRI, Sociedad de Promoción y Reconversión Industrial creada el año anterior por el Departamento de Industria y Energía del Gobierno Vasco. Es un proyecto dirigido a mejorar la infraestructura de la industria vasca, proporcionando una oferta de edificios industriales que faciliten el desarrollo y tejido productivo. Los destinatarios de este programa son pequeñas y medianas empresas, sobre todo las de nueva creación, las innovadoras y aquéllas que precisen trasladarse por ampliación. Las industrialdeas pretenden un asentamiento adecuado de la actividad industrial que favorezca una ordenación del territorio equilibrada. Se aspira a que la instalación de las empresas en las industrialdeas mejore su imagen al encontrarse en un polígono industrial urbanizado de acuerdo a los parámetros actuales. Son polígonos proyectados de forma modular, lo que permite adaptarse a las distintas necesidades de las diversas empresas. Están construidos siguiendo modernas técnicas de aislamiento y aprovechamiento luminoso que proporcionan un mayor ahorro energético y confort. El esmerado diseño pretende mejorar la productividad de la industria local ofreciendo unos edificios adecuados para el desarrollo de la actividad.

Por otro lado, el alto coste de este tipo de instalaciones no sería accesible para las PYMES, sobre todo si son de nueva creación, ya que precisan centrar la inversión en la actividad que inician. Por ello se ha establecido que el precio sea inferior al del mercado (un 20 o $30 \%$ ) y ofrecen facilidades de pago para acabar adquiriendo el local mediante un contrato de arrendamiento con opción de compra. Los criterios de localización son muy diversos, desde la creación de actividad industrial en áreas carentes de tradición, la revitalización de una comarca deprimida hasta la complementariedad con otras iniciativas públicas o privadas.

El desarrollo, funcionamiento y organización de las industrialdeas está sometido a un protocolo de actuación que prácticamente se repite en todos los ejemplos. Así, si el SPRI colabora en la financiación, coordinación y asesoramiento de las distintas industrialdeas creadas, cada una de ellas presenta un funcionamiento autónomo. En este sentido, se apuesta por la creación de Sociedades Participativas de la Sociedad Pública SPRI, y son declaradas como Sociedades Públicas integrantes de la Administración Institucional de la Comunidad Autónoma de Euskadi².

1 Como señala Torres, «tienden a proliferar los soportes flexibles de apoyo a las empresas con actuaciones encaminadas a incidir en el contexto en el que se desenvuelve la actividad productiva (difusión y producción de la tecnología, formación de mano de obra, estímulos a la innovación, promoción de nuevos mercados, divulgación de técnicas de gestión y organización empresarial, etc.) (1995, p. 229)».

2 Se regulan a partir del artículo 19 del Decreto legislativo 1/1988 de 17 de Mayo, por el que se aprueba el texto refundido de las disposiciones legales vigentes sobre Principios de Ordenadores de la Hacienda General del País Vasco, en el que se establece que son Sociedades Públicas en cuyo capital sea mayoritaria la participación de la Administración de la Comunidad Autónoma de Euskadi o de sus Entes Institucionales, ya se rijan éstos por derecho público o privado. 
Las entidades mercantiles se generan con un capital social aportado por accionistas que ostenten el carácter de entes u organismos de la administración pública, autónoma o local, Cajas de Ahorro de la Comunidad Autónoma o entes o sociedades constituidos total o parcialmente por los entes administrativos o financieros anteriormente citados. Lo habitual es que sea la sociedad SPRI, como representante del Gobierno Vasco, la Diputación Foral correspondiente y el Ayuntamiento implicado los que aporten el capital social de la entidad mercantil. Por lo demás, estas sociedades están regidas por una junta general y un consejo de administración, que elige en su seno a su presidente y secretario, siguiendo el sistema habitual de las sociedades mercantiles.

El objeto de las sociedades mercantiles creadas es el de estimular y promover la iniciativa y la inversión industrial en el término municipal en el que se ubican, mediante la construcción de complejos de pabellones industriales y edificaciones complementarias, así como su gestión hasta la total transferencia de la titularidad de los mismos.

Una de sus principales labores, por tanto, es la de calificar y promover suelo industrial de iniciativa pública. En estos casos, es el Ayuntamiento el que toma la iniciativa de calificar suelo para usos industriales y establece un sistema de acuerdo-compensación a los propietarios o, de no haber acuerdo, recurre a la expropiación.

Una vez obtenida la propiedad del suelo, el Ayuntamiento requiere la colaboración de la Sociedad Mercantil creada para gestionar el suelo industrial generado y, fundamentándose en la Ley 20/1998, de Patrimonios Públicos de Suelo, que autoriza la cesión de bienes de patrimonio municipal del suelo a entes instrumentales cuando su destino sea la promoción pública de actividades económicas, cede directamente el suelo de su propiedad.

A su vez, la sociedad mercantil, como destinataria del suelo, asume por su parte la urbanización del área, la construcción del polígono y la promoción de las naves resultantes. Del mismo modo, en la medida en que es la destinataria final del suelo, asume compensar a los accionistas, generalmente el ayuntamiento y el SPRI, de los gastos realizados en la gestión urbanística. Por tanto, compete a la sociedad que regula el área industrial creada determinar los precios y condiciones de venta de los solares resultantes de la actuación con criterio de recuperación de las cantidades económicas invertidas en la actuación, más los costes de estructura imputables. La práctica habitual es la de reducir los precios como medida de promoción y que sean las entidades públicas las que asuman la deuda originada ${ }^{3}$.

De este modo, la Sociedad Mercantil ofrece edificios industriales en polígonos adecuadamente urbanizados y comunicados, cuyo diseño y construcción posibilita ubicar en su seno pequeñas y medianas empresas industriales, y en especial aquellas de nueva creación que por razón de su crecimiento o inadecuado asentamiento deban de ser trasladadas. Y lo hace ofreciendo a las empresas el acceso a la propiedad inmobiliaria a un coste atractivo. La práctica habitual es la de ofrecer el pabellón construido aunque, en determinados casos, se ofrece la parcela urbanizada pero sin la ejecución del polígono.

Por tanto, el destino final de los polígonos creados es la venta a particulares aunque en este proceso generalmente se ofrecen facilidades para su adquisición en un periodo prolongado, generalmente de diez años. Por lo general, las Sociedades Mercantiles se reservan el derecho a compra en relación al precio original de venta en aquellos casos en los que el particular decide vender la propiedad, normalmente durante un periodo de diez años.

3 Como se recoge en el convenio de promoción de suelo industrial en Irun «Estos precios prodrán ser minorados, como medio de promoción, o aumentados, para compensar anteriores rebajas, de modo que la recuperación final resulte equilibrada». (Convenio de promoción del suelo industrial de Arretxe-Ugalde. 11-1-1999). 
Una vez iniciada la actividad en los polígonos industriales son los usuarios los que gestionan el mantenimiento de los elementos comunes de la industrialdea y lo hacen creando una comunidad de propietarios que gestiona desde la recogida de basura hasta el aparcamiento, que elige un Junta de Propietarios y que abona unas tasas establecidas.

Por lo tanto, se define un modelo unitario, fundamentado en la colaboración entre las distintas administraciones públicas, de la escala autonómica a la local, y apoyado en una sociedad de promoción industrial y sus filiales con el fin de impulsar la iniciativa privada en el desarrollo industrial. En este proceso se pasa de la intervención pública a la gestión y propiedad privada tutelada por la administración.

\section{Agroaldea: de la visión industrial al mundo agrario}

Retomando la concepción de la industrialdea nace su homónimo agrario, constituido por un polígono de parcelas dedicadas generalmente a producción hortícola y disfrutadas por nuevos horticultores o agricultores que por diferentes razones necesitan trasladar su explotación a mejores ubicaciones. La coincidencia de los objetivos no supone, sin embargo, una asimilación de la estructura organizativa que el polígono industrial tiene en la economía y sociedad vasca. A continuación vamos a aproximarnos a las características, organización y extensión de una iniciativa original.

\subsection{Agroaldea como polígono hortícola}

El País Vasco-Atlántico forma en su conjunto una gran región urbana. La ciudad se extiende por todas sus comarcas urbanizando la mayoría de sus valles. Las actividades agrícolas quedan arrinconadas a zonas más alejadas, de mayor altura y pendiente, limitando sus usos a aprovechamientos ganaderos, a menudo extensivos.

El espacio físico y las funciones urbanas reducen los suelos arables. El desarrollo de la horticultura, necesitada de parcelas relativamente llanas, espacios que, en un medio de montaña tan sólo se desarrollan en los fondos de los valles, queda limitado ante el avance de la ciudad.

Las soluciones espaciales que las distintas instituciones públicas y agencias de desarrollo rural dan al problema de la falta de suelo hortícola es, cuanto menos, original. El concepto de Agroaldea se aplica en el País Vasco a polígonos de parcelas acondicionados para el uso de actividades agrícolas, preferiblemente de titularidad pública y con una dotación urbanística común, cuya característica principal es su puesta a disposición de los agricultores demandantes bajo arrendamientos de cuantía normalmente simbólica.

«Una agroaldea viene a constituir un polígono agrícola y, aunque en el País Vasco no existen antecedentes en este sector, la idea surge de lo que en el caso del sector industrial hemos denominado "industrialdea", es decir, pequeños polígonos industriales dotados de servicios comunes y promovidos por instituciones públicas (Gobierno Vasco, Diputación, Ayuntamientos), que ponen al servicio de pequeñas empresas terrenos, infraestructuras e instalaciones necesarias para su normal funcionamiento. En este centro, se agrupan una serie de productores y empresas agrarias que compartirán, además del terreno de propiedad pública, una infraestructura planificada y una serie de servicios comunes determinados y gestionados por ellos mismos» (HAGINA, nº 4, 1995, p. 15).

La fuerte presión urbana ejercida sobre el suelo rural, que impide crear nuevas explotaciones hortícolas o ampliar las existentes, es la razón que justifica la aparición de este concepto. Su materialización no es original y en comunidades próximas, como Navarra, hay varios modelos de este tipo de espacios. La novedad estriba en que no son nuevos produc- 
tores los que acceden a estas parcelas sino también agricultores ya consagrados que necesitan terreno para mantener o ampliar su cultivo. La presión existente en esta zona justifica que estas iniciativas estén dirigidas por y para esta tipología de horticultores.

El origen de estas Agroaldeas viene provocado, por tanto, por la creciente demanda de suelo por parte de agricultores profesionales que desean trasladar, reubicar o ampliar sus explotaciones o nuevos agricultores que deseen instalarse en el sector ${ }^{4}$. En el País Vasco, estas modalidades se desarrollan preferentemente en el periurbano de San Sebastián. En este caso, la demanda de tierras se ve aumentada por el importante desarrollo urbanístico y de infraestructuras, lo que provoca que un gran número de agricultores sean desalojados de sus tierras por expropiación o, simplemente, no puedan acceder a la propiedad del suelo ante las expectativas urbanísticas suscitadas en el espacio rural ${ }^{5}$.

La titularidad pública, deseable para el desarrollo de proyectos de este tipo, se ve limitada muchas veces por el escaso suelo de calidad agrológica aceptable que se encuentra en manos de las administraciones, lo que obliga al desarrollo de estas iniciativas en suelos con limitaciones en algunos casos severas, que lleva a realizar importantes actuaciones urbanísticas para el acondicionamiento de estas superficies hortícolas.

\subsection{Diferentes modelos para una misma iniciativa}

Las Agroaldeas en funcionamiento se corresponden en todos los casos con polígono de parcelas agrícolas de cultivo intensivo profesional. Este modelo es el utilizado en las de San Marcos (Errenderia-Donostia), Asteasu, Arragua (Oiartzun), Astigarraga y Lezo. Consiste en polígonos de propiedad pública, divididos en parcelas de entre 4.000 y $10.000 \mathrm{~m}^{2}$, con infraestructuras generales comunes (luz, agua, accesos, cierres) y destinadas al cultivo de hortalizas, plantas o flor cortada, tanto en cultivo directo sobre el suelo, como en hidroponía o maceta y en invernaderos o al aire libre. En este momento se encuentran en diversos grados de desarrollo agroaldeas que responderían al mismo modelo y fines (Bergara, Irun, Astigarraga) o con fines de provisión de planta a productores ecológicos (Agroaldea ecológica de Billabona).

Profundizando en algunas de las particularidades de las agroaldeas en funcionamiento cabe señalar como cada una aprovecha las oportunidades que le brinda el entorno. En el caso de la agroaldea de San Marcos, las cuatro parcelas situadas en el mismo tienen la posibilidad de acceder a una fuente de calor gratuita, como es el agua de refrigeración de los motores de la planta de biogás del vertedero comarcal ${ }^{6}$. A su vez la Agroaldea de Oiartzun

4 Para más información sobre el concepto y el funcionamiento de las agroaldeas ver HAGINA, ${ }^{\circ} 4,1995$, pp. $15-16$; n 12,1997 , pp. 36-41; nº 19, 1998, pp. 46-47; n 20, 1999, pp. 42-44.

5 Demanda que queda reflejada en la proliferación de pequeñas parcelas cultivadas fuera de toda ordenación urbanística, coincidiendo con fenómenos propios de áreas de gran concentración de población. En este sentido COLMENAR lo consideraba como «un fenómeno que estaba apareciendo en esa época en el área metropolitana de Madrid: los huertos ilegales, los huertos en precario que surgían en muchos terrenos públicos no vigilados, como los cercanos a RENFE o los próximos al aereopuerto» (1988, p. 61).

6 El vertedero de San Marcos posee un sistema de captación del biogás originado por la fermentación de los residuos, que es recogido y trasladado a una planta de tratamiento donde unos motores lo utilizan como combustible para mover un alternador que genera energía eléctrica. En la refrigeración de estos motores se emplea enormes cantidades de agua que, una vez que alcanzan la temperatura de $80^{\circ} \mathrm{C}$, son enviadas a unas torres de refrigeración en las que se vuelven a enfriar hasta alcanzar de nuevo una temperatura inferior a $30^{\circ} \mathrm{C}$. Con la incorporación de los invernaderos, ahora este agua caliente es enviada directamente a los invernaderos mediante un equipo de bombeo. Allí recircula hasta perder calor y, tras ello, vuelve a la planta de transformación de biogás a una temperatura próxima a $30^{\circ} \mathrm{C}$. El sistema de calefacción, una vez que se ha acometido la inversión, es casi gratuito, con la excepción del costo en electricidad que supone el bombeo del agua desde las torres actuales hasta los invernaderos, situados a una cota aproximada de $30 \mathrm{~m}$. por encima de la planta (ALBERDI, 1999, p. 44). 


\section{Agroaldeas en la Comunidad Autónoma del País Vasco. Año 2004}

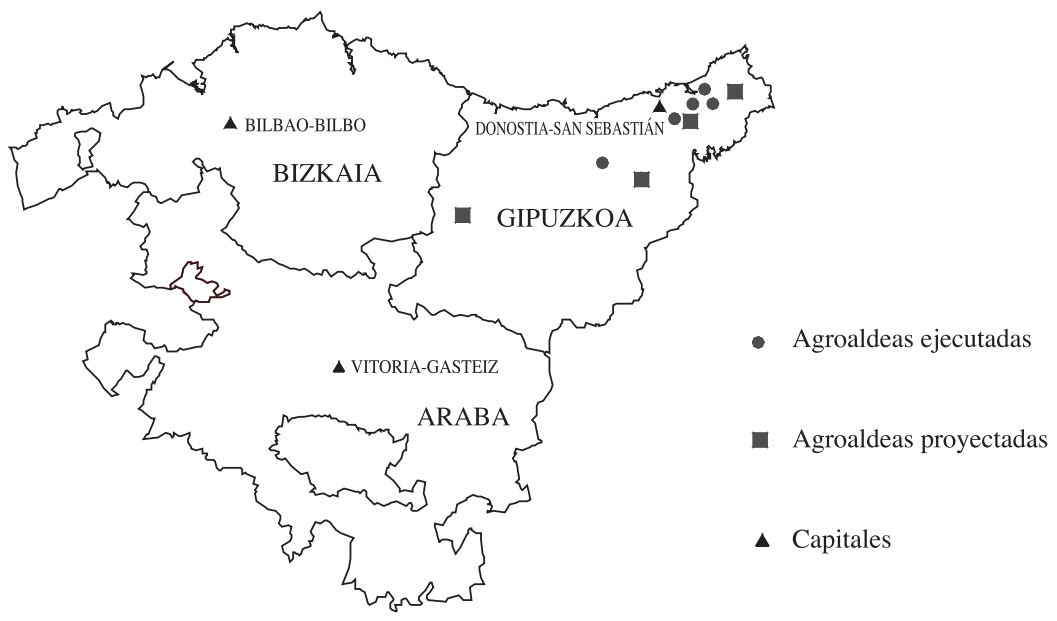

Fuente: Udalplan 2003. Elaboración propia.

cuenta con una planta de cogeneración eléctrica mediante gas natural que calefacta los invernaderos mediante la recirculación del agua de refrigeración de los motores. Se prevé que la futura Agroaldea de Bergara pueda también aprovechar el circuito de refrigeración de la planta de Sidenor ubicada en las proximidades.

En relación al tipo de terreno que utilizan, estas agroaldeas se asientan bien sobre tierras cultivables de mayor o menor valor agrológico (Asteasu, Astigarraga, Lezo) o bien sobre terrenos baldíos, como pueden ser rellenos de vaguadas (Oiartzun) o áreas recuperadas de vertederos (San Marcos). En este último caso la instalación de actividades basadas en los cultivos sin suelo, hidroponía o maceta, se hace imprescindible por el nulo valor agrícola o la toxicidad del suelo soporte, además de implicar la utilización de zapatas corridas de hormigón, para la sujeción de los invernaderos, con el fin de evitar fracturas estructurales por hundimientos del suelo. A pesar de estos inconvenientes estas parcelas resultan de interés, además de por sus posibilidades energéticas, por su escaso valor para otras actividades productivas, por lo que la competencia de aprovechamiento es menor que en otros suelos. Realizamos a continuación una breve descripción de los polígonos agrícolas materializados hasta el momento.

- Agroaldea de San Marcos (Errenderia-Donostia). Se trata de un polígono de cuatro parcelas, de alrededor de $29.000 \mathrm{~m}^{2}$ de extensión total, sobre una de las plataformas de relleno del Vertedero de R.S.U. de San Sebastián y que da servicio alrededor de 360.000 personas. La agroaldea ha sido impulsada por la Asociación de Desarrollo Rural comar$\mathrm{cal}^{7}$ y el Ente Vasco de la Energía. Gracias a la colaboración de esta entidad se puede uti-

7 Las Asociaciones de Desarrollo Rural surgen al amparo de la Ley de Desarrollo Rural del País Vasco de 1998. En ella se establecen los órganos que han de cumplir la función de coordinar y dirigir las actuaciones en 
lizar como calefacción el agua de refrigeración de los motores de la planta de biogás del vertedero. En todos los casos son agricultores ya asentados en el sector.

- Agroaldea de Arragua (Oiartzun). La primera de este tipo en entrar en funcionamiento, consta de cinco parcelas contiguas, con una extensión que ronda entre $5.000 \mathrm{y}$ $7.000 \mathrm{~m}^{2}$, dedicadas a la producción en invernaderos de tomate, flor cortada y planta ornamental y en un caso a la producción de planta ornamental y forestal con destino a recuperaciones paisajísticas y jardinería, producción que también se realiza en el exterior. Los cultivos de invernadero se han desarrollado en un primer momento en cultivos sobre el suelo, pero comienza a imponerse la hidroponía, o cultivo en sacos de perlita, y el cultivo en mesas o macetas ${ }^{8}$. Dispone en las inmediaciones de una planta de cogeneración energética gestionada por Guascor, que suministra agua caliente para calefacción a las explotaciones del agroaldea. La titularidad de las parcelas corresponde al Ayuntamiento de Oiartzun y los usuarios son agricultores de nueva instalación en tres de las cinco explotaciones que se han ubicado en el lugar.

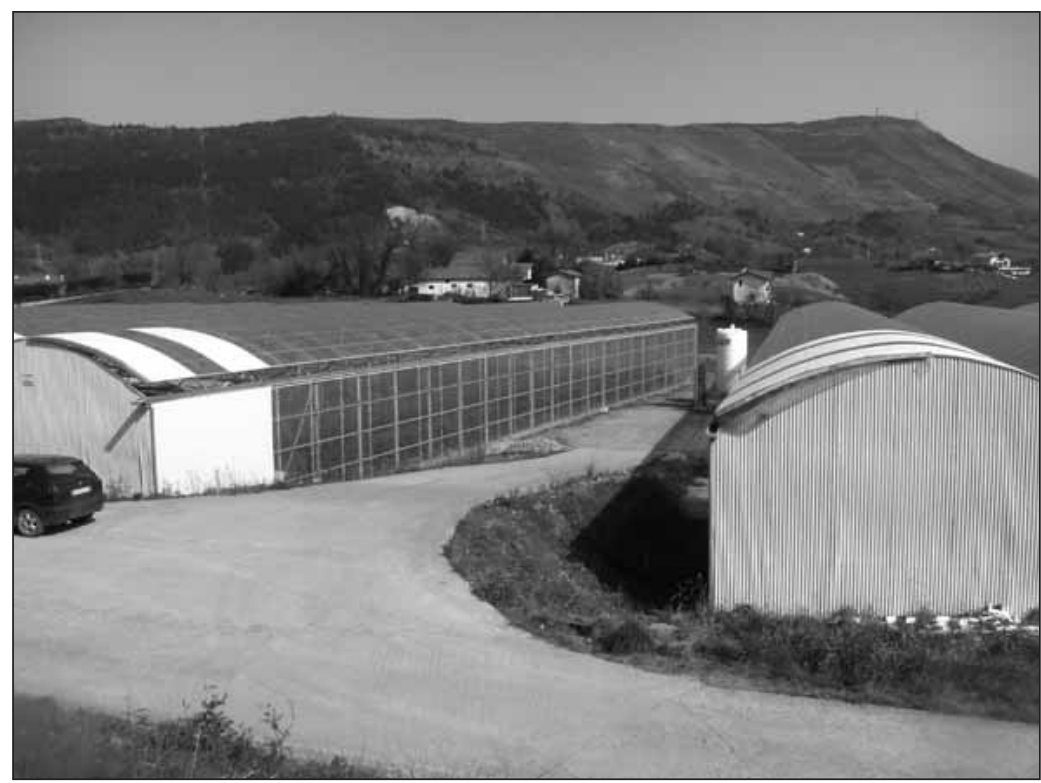

FIgURA 1. Agroaldea de Oiartzun. Año 2004.

materia de desarrollo rural, y entre éstos la aplicación de los programas diseñados en cada comarca se decide que sea realizada por órganos locales. En este sentido, se apuesta por la creación de una nueva figura, las Asociaciones de Desarrollo Rural, que se establecen como instrumento de participación y colaboración de los agentes económi$\cos$ y sociales en las actuaciones de desarrollo rural. La Ley establece los requisitos para el reconocimiento de las ADR con el carácter de entidades de derecho privado y representativas de los diferentes sectores económicos y sociales de la zona comprendida en un PDR. Estas Asociaciones vienen a dar continuidad a las anteriores Asociaciones de Agricultura de Montaña o Agencias de desarrollo rural que ya existían en las diferentes comarcas y que se ven ahora reforzadas con la asunción de nuevas competencias otorgadas con la aprobación de los PDR comarcales y con su designación como responsables de la ejecución de sus contenidos.

8 Según señala PEREZ, gerente de ASTHOR-AGRICOLA «la tendencia actual entre los profesionales es la realización de instalaciones del tipo multicapilla (placa) aunque aquellas personas que acceden a trabajar en el sector agrícola por vez primera montan por lo general túneles. Y son éstos precisamente los que predominan en la Cornisa» (HAGINA, nº 13, 1997, p. 23). 
- Agroaldea de Lezo. Se sitúa en la vaguada de Antzillastegi, cercana al Alto de Gaintxurizketa y con aproximadamente $15.440 \mathrm{~m}^{2}$ en bancales, habiéndose presentado para el aprovechamiento de la misma un proyecto conjunto por parte de dos agricultores con producción de árboles y arbustos autóctonos destinados a obras de recuperación medioambiental y producción de flor y planta en maceta. En ambos casos son nuevos agricultores.

- Agroaldea de Astigarraga. Propiedad del Ayuntamiento de Donostia pero situada en el municipio de Astigarraga, dispone de $14.780 \mathrm{~m}^{2}$ de parcela dentro de una finca de mayor tamaño cedida a su vez a Talleres Protegidos Gureak para la producción de flor y planta ornamental. Se destina fundamentalmente a proporcionar tierra a agricultores desalojados por las obra del Segundo Cinturón Viario de Donostia. En la actualidad se han instalado cuatro explotaciones, dos con producción de tomate y pimiento y una más dedicada a la producción de flor cortada. En las inmediaciones de esta Agroaldea se prevé la implantación de una segunda de aproximadamente $60.000 \mathrm{~m}^{2}$, en la explanada de relleno que van a producir las obras de la autovía del Segundo Cinturón de San Sebastián.

- Agroaldea de Asteasu. Se ubica en una zona de vega fluvial de $19.082 \mathrm{~m}^{2}$, disponiendo de siete parcelas de $2.726 \mathrm{~m}^{2}$. En este momento se encuentran adjudicadas cinco de las parcelas, destinadas a la producción hortícola, tanto en cultivos con suelo como en hidroponía. El desarrollo de esta Agroaldea se impulsó mediante un Convenio de Cooperación firmado entre el Ayuntamiento de Asteasu y la Diputación Foral de Gipuzkoa.

Los modelos de cesión o arrendamiento de las parcelas situadas en los agroaldeas mencionadas han sido variados. En algúnos, la selección y adjudicación de las parcelas se ha realizado directamente entre la administración implicada (Ayuntamiento) y el usuario parti-

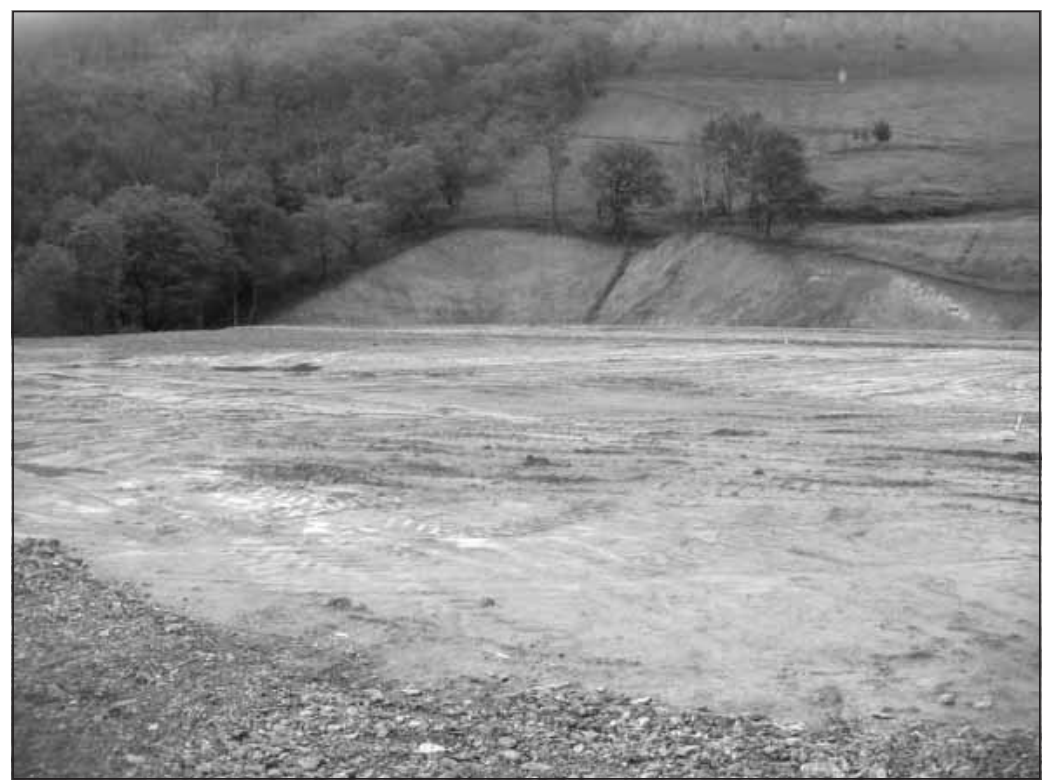

FIgUra 2. Obras de Acondicionamiento del Agroaldea de Astigarraga. Año 2004. 
cular, en otras la adjudicación se a efectuado a favor de una asociación de productores o de la Asociación de Desarrollo Rural Comarcal y en todos los casos, a pesar de ser la administración titular de la finca la que cumplimentaba el contrato, se ha contado con la colaboración de la correspondiente Asociación de Desarrollo Rural para el desarrollo y financiación del proyecto, urbanización de las parcelas, la selección de los adjudicatarios y el control de la evolución de las instalaciones.

Se establecen unas condiciones de uso comunes, destinadas a regular el correcto funcionamiento de la utilización de la parcela. Las más habituales de las condiciones establecidas son:

- Conservación de las instalaciones y el entorno.

- Compromiso de mantenimiento de la actividad agraria.

- Imposibilidad de subarrendar ni ceder el uso de la parcela.

- Dedicación exclusiva de la parcela a la finalidad admitida, esto es la producción hortoflorícola.

- Prohibición de ejecución de obras e instalaciones sin previa autorización del titular de la finca.

- Exoneración de responsabilidad del titular de la finca frente a cualquier actuación gravosa del usuario.

- Estipulación de una renta, generalmente simbólica, a abonar en un único plazo anual.

Partiendo de los condicionantes comunes profundizamos a continuación en las pecularidades que cada una de las cesiones utilizadas presenta, agrupándolas en tres tipologías distintas.

- Contrato de Concesión, salvo derecho de propiedad y sin perjuicio de terceros. Modelo utilizado en los Agroaldeas de San Marcos (Errenderia-Donostia) y Antzillastegi (Lezo). En el caso de la Agroaldea de San Marcos, se materializó mediante un pliego de cláusulas administrativas, por el que se realizó un concurso mediante procedimiento abierto, en el que se recogieron propuestas de uso, seleccionándose la promovida por la Asociación de Productores del Agroaldea de San Marcos, con los que la Mancomunidad formalizó el Contrato Administrativo de Concesión de disfrute y aprovechamiento privado de bienes de dominio público. El Contrato de Concesión se estipulaba por un periodo de 15 años, con la posibilidad de prórrogas anuales hasta un máximo total de 20 años de concesión. En el caso de Antzillastegi, el Contrato de Concesión tuvo por adjudicatario a la Asociación de Desarrollo Rural de Donostialdea-Bidasoa (Behemendi), por un plazo improrrogable de 20 años y con el fin de destinar la Agroaldea a promocionar actividades agrícolas. Esta Asociación, posteriormente, adjudica, mediante concurso de proyectos, las parcelas que el Ayuntamiento le ha cedido para su explotación.

- Contrato de Arrendamiento. Utilizado en Asteasu y Arragua (Oiartzun), por el que se formaliza un contrato de arrendamiento directamente entre el Ayuntamiento de la localidad y el usuario de cada parcela. En el caso de Arragua el arrendamiento se realiza para un plazo de 15 años prorrogables durante quince más. Estableciendo un arrendamiento revalorizable anualmente en función del incremento del I.P.C. En cuanto al Agroaldea de Asteasu los plazos del arrendamiento son de 12 años para los solicitantes que realizan inversiones superiores a 3.000 euros (todos aquellos que implantan invernaderos) y de tres años para los que realizan inversiones de inferior cuantía (usualmente aquellos dedicados a la producción al aire libre). Estos plazos se 
suponen prorrogables, en caso de mutuo acuerdo y durante el periodo que se quiera establecer, siendo en todo caso el periodo máximo de arrendamiento de 20 años. En esta última Agroaldea, la D.F.G. participa en la mesa de contratación junto al Ayuntamiento de la localidad y la Asociación de Desarrollo Rural de Tolosaldea, que a su vez es la encargada de recoger las solicitudes.

- Acuerdo de Cesión de Uso. Empleado en la Agroaldea de Astigarraga, en el que el Ayuntamiento de Donostia, propietario de la finca cedió el uso de $14.780 \mathrm{~m}^{2}$ de la misma con el fin de implantar un Agroaldea que sirviera de reubicación a afectados por las obras de infraestructura de la comarca. La cesión se realizó a favor de la Asociación de Desarrollo Rural de Donostialdea-Bidasoa, por un periodo de 15 años, prorrogables previa solicitud y nuevo acuerdo municipal. El protocolo que ejecuta posteriormente esta Asociación es similar al utilizado en Lezo, es decir, ofrecer las parcelas mediante concurso a particulares para su explotación. Las condiciones exigidas por el ayuntamiento son respetadas por esta Asociación e impuestas a los usuarios de las parcelas.

\section{Una modalidad no exenta de problemas: debilidad del agroaldea frente al indus- trialdea}

Desde el año 1996 en el que empiezan a desarrollarse los trabajos de impulso de la primera Agroaldea en Gipuzkoa se ha conseguido que sean varios los proyectos ejecutados y que se este planeando la posibilidad de desarrollar nuevos agroaldeas en Bentas (Irun), Astigarraga, Billabona y Bergara. Los datos resumidos del impacto de esta política no deja lugar a dudas del progreso de la misma:

- Cinco Agroaldeas en funcionamiento: Arragua (Oiartzun), San Markos (ErrenteriaDonostia), Antzillastegi (Lezo), Astigarraga y Asteasu.

- Cuatro Agroaldeas en fase de proyecto: Bentas (Irun), Astigarraga, Billabona y Bergara.

- Ampliación de 9 explotaciones preexistentes.

- Creación de 6 nuevas explotaciones.

- Implantación de $39.900 \mathrm{~m}^{2}$ de invernaderos.

- Desarrollo de $15.500 \mathrm{~m}^{2}$ de explotación al aire libre.

- Creación de 32 puestos de trabajo fijos y otros tantos temporales.

Debemos tener en cuenta que estamos ante un subsector con escasa representación en la Comunidad Autónoma, en la que apenas un centenar tiene la consideración de explotación exclusiva. El impacto de las producciones obtenidas en las agroaldeas, y más tratándose generalmente de instalaciones modernas y de alta productividad, es notorio especialmente en el territorio en el que se ubican, Gipuzkoa'.

El resultado satisfactorio del sistema de Agroaldea, sin embargo, está en este momento en entredicho debido a factores que prueban la debilidad del modelo de desarrollo elegido.

9 Según señala IKT (2003), la horticultura ha sido una actividad tradicional en el caserío de Gipuzkoa aunque generalmente ha ocupado un lugar secundario tanto económica como espacialmente. Las únicas zonas en las que la huerta tenía cierta importancia coincidían con aquellas áreas más próximas a los núcleos urbanos de la provincia, especialmente San Sebastián y sus alrededores, donde se combinaba con una función ganadera. Como consecuencia, son muy pocas las unidades con una orientación hortícola. Tan sólo el $4 \%$ presentan una OTE en horticultura en el territorio de Gipuzkoa y de ellas sólo 32 (6.4\%) superan la segunda UTA, a partir de la cual se equipara con una actividad profesional. 
Una muestra de ello son los problemas de continuidad que presentan actualmente las agroaldeas de Arragua y Asteasu. En el primer ejemplo, el Departamento de Carreteras de la D.F.G. proyecta una nueva carretera que atraviesa la Agroaldea. En caso de aprobarse esta propuesta se pasaría a expropiar los citados terrenos, actuación que aunque conlleva la indemnización de inversiones realizadas en mejoras productivas, supone la practica desaparición de todas ellas, pues su traslado dentro de la comarca se vería casi imposibilitado por el alto precio del suelo ${ }^{10}$.

Por otro lado, en el caso de Asteasu, el Ayuntamiento quiere implantar en la localidad una nueva planta industrial de la empresa Gamesa, para lo que pretende recalificar el suelo ocupado por la Agroaldea. De esta forma, ha comenzado a no renovar los contratos trianuales firmados con algunos productores y ha suspendido la firma de dos nuevos contratos.

Estas proyecciones sobre las Agroaldeas tienen además del impacto sobre las explotaciones afectadas, un efecto disuasorio sobre la demanda de parcelas en otras iniciativas, pues los inversores (que en algunos casos deben realizar inversiones superiores a los $180.000 €$ ) dudan a la hora de promover una explotación con una protección jurídica débil y pendientes de la voluntad cambiante de las distintas administraciones. Además, estas actuaciones también pueden llegar a desmoralizar a las entidades impulsoras de Agroaldeas, pues proyectos que conllevan un fuerte trabajo de gestión y conciliación de intereses se ven abocados al fracaso a los pocos años de desarrollarse con éxito.

Otro aspecto que queda en evidencia es la falta de un protocolo de actuación común en la puesta en marcha de las agroaldeas. Hasta el momento no ha habido una apuesta pública decidida por esta modalidad de desarrollo agrario sino la iniciativa de una serie de Asociaciones de Desarrollo Rural que, atendiendo a las circunstancias de cada una de las agroaldeas y al apoyo puntual de la Diputación Foral, han dado inicio a toda una serie de proyectos siguiendo un patrón más o menos común.

La inexistencia de una política decidida e impulsada desde las administraciones públicas los polígonos hortícolas limita sus posibilidades de desarrollo. Como consecuencia, son terrenos marginales los que en mayor medida son utilizados, suelos sobre los que no planean otro tipo de iniciativas y que las entidades municipales ceden a las Asociaciones de Desarrollo Rural sin exigir retribuciones a cambio. La falta de una entidad dirigida a promover la gestión del suelo rural dificulta la adquisición de otro tipo de suelos, limita la llegada de ayudas económicas e incluso provoca que otras instituciones o departamentos ignoren la labor de estos espacios frente a otras necesidades dotaciones o de promoción económica.

La comparación del modelo de funcionamiento de las agroaldeas con el de las industrialdeas deja en evidencia la falta de concreción y la debilidad de las primeras, aspecto que finalmente se traduce en los problemas que atraviesan algunos de los polígonos hortícolas ya iniciados, situación que no se repite en el caso de las industrialdeas.

La razón de la fortaleza de los polígonos industriales de promoción pública estriba en la estructuración que define su funcionamiento, con una sociedad mercantil financiada desde el Gobierno Vasco, con recursos económicos y personales, con posibilidad de intervenir en procesos de compra de suelo y de expropiación y capaz de organizar toda una serie de unidades individuales, sociedades mercantiles que dirigen el funcionamiento de los polígonos a nivel local o en todo caso comarcal, que aglutinan recursos municipales y provinciales y

10 La compra de suelo para promociones de vivienda privadas que se están realizando en la proximidad de San Sebastián, dirigidas a la construcción de bloques residenciales, ha alcanzado a principios del año 2004 la cifra de $360 € / \mathrm{m} .2$ Ante unos precios de tal dimensión las expectativas generadas imposibilitan cualquier compra de suelo para una utilidad agraria en un área muy urbanizada. 
que ejecutan, dirigen y gestionan las estructuras productivas creadas. Es un modelo estructurado, en el que toman parte las distintas instituciones de la Comunidad Autónoma, desde el Gobierno hasta el Ayuntamiento, que aprovecha las competencias de cada una, tanto en materia presupuestaria como en posibilidades de ordenación y con un fin definido, la venta a un precio atrayente de las estructuras productivas originadas. La participación de toda la estructura pública y la importancia de las operaciones generadas dan a este modelo un reconocimiento económico y social y un respeto a sus actuaciones por parte de otros departamentos que reconocen estas estructuras en sus planificaciones.

El modelo planteado en las Agroaldeas, aún siendo una continuidad del impulsado por las industrialdeas, presenta unas carencias organizativas y una falta de reconocimiento político que le aleja de la efectividad y posibilidades de desarrollo de su homónimo industrial. La principal insuficiencia que presenta es la inexistencia de un esquema organizativo para toda la Comunidad Autónoma Vasca. A diferencia de las industrialdeas no existe en el ámbito agrario una institución que promocione iniciativas dirigidas a retener suelo para la promoción de actividades agrarias, ni siquiera aquéllos que pueden quedar libres como consecuencia de la cesión de personas que se acogen a programas de prejubilación ${ }^{11}$. En la medida en que no hay una entidad dirigida a gestionar suelo agrario se hace difícil promocionar iniciativas de desarrollo que se fundamenten en este recurso, se carece de una entidad que defienda los intereses agrarios ante otros departamentos e instituciones y se dificulta captar recursos económicos para poner en marcha este tipo de iniciativas. Si a ello añadimos que la finalidad de las agroaldeas no es la de vender el terreno sino la de promocionar una función apoyando la incorporación de agricultores que por falta de recursos tienen dificultades de acceder al suelo, los rendimientos económicos que origina para las entidades económicas implicadas son nulos y el número de proyectos iniciados muy reducido. Como consecuencia, a pesar de que hace más de una década que se materializó la primera agroaldea su expansión apenas ha superado el ámbito comarcal del área de San Sebastián.

La inexistencia de una entidad de gestión de suelo rural deja en manos de las Asociaciones comarcales la promoción de los polígonos hortícolas. Estas entidades, de naturaleza privada pero que funcionan con recursos públicos, recurren a la colaboración de los ayuntamientos para reutilizar suelo de su propiedad para este tipo de uso. Con los fondos con los que cuentan realizan la adecuación de las parcelas y colaboran en la instalación de los agricultores. La propiedad del suelo, sin embargo, continua siendo municipal y el ayuntamiento se reserva la opción de recuperar dichos suelos si los necesitara para otras funciones. El mantenimiento de la agroaldea depende a menudo de la voluntad política y de los intereses que en cada momento tenga el ayuntamiento que, o por necesidades de equipamiento o por otras expectativas económicas, puede decidir compensar a los horticultores y recuperar las parcelas para otros usos. La inexistencia de una sociedad que compre suelo resta estabilidad a muchos de estos proyectos.

Otro aspecto relevante que origina la falta de una entidad de promoción agraria estriba en la pérdida de oportunidades de hacerse con suelo útil originado como consecuencia de las obras de infraestructura que se realizan en un medio de montaña, como es el área vasco-

11 Las únicas iniciativas de promoción de suelo agrario en España están ligadas al abandono de la actividad por agricultores de edad avanzada e incluso en este caso es muy difícil poner en marcha en todo el País VascoAtlántico iniciativas similares, por ejemplo, a las que se recogen en la Ley 4/1989 del Principado de Asturias, por medio de la cual se crea el Banco de Tierras. Tan sólo el disponer de una base patrimonial pública lo suficientemente amplia permitiría disponer de un patrimonio mínimo. El precio de la tierra es excesivamente elevado y la presión tan importante que difícilmente se puede acceder a otro tipo de terrenos. De hecho, es esta Comunidad la que encabezaba en el año 2000 el número de agricultores acogidos a los programas de prejubilación (CINCO DÍAS, 2001). 
atlántica del País Vasco. Son muchos los rellenos, vertederos y nuevos espacios originados por estas obras y que, por la inexistencia de una entidad pública que recupere este suelo, compense a sus propietarios por el nuevo uso que se va a instalar e inicie un proceso de adecuación para la materialización de polígonos agrarios, se permite al anterior propietario ejercer el derecho a reversión y retener antiguos terrenos baldíos reconvertidos ahora en suelos llanos y útiles para otros usos.

A todo ello hemos de añadir que pretendemos desarrollar estos modelos en un área en la que el suelo útil es muy escaso y en la que las necesidades urbanas e industriales priman sobre otro tipo de demandas. El peso de la agricultura es muy reducido, sus necesidades de suelo son importantes y, ante posibles conflictos espaciales, la actividad primaria es la que es arrinconada ${ }^{12}$.

No hay, al menos por el momento, una apuesta decidida por potenciar un modelo espacial para el desarrollo de la horticultura vasca y la incorporación de nuevos agricultores. La falta de una entidad de gestión de suelo rural para el conjunto de la Comunidad dificulta la materialización de un modelo que se ha mostrado adecuado para desarrollar un subsector, el hortícola y que está presentando esta última década las mayores cotas de crecimiento y de incorporación de jóvenes agricultores, tal y como lo recoge el Plan de Jóvenes Agricultores de la Comunidad Autónoma Vasca (1993).

\section{Un futuro que pasa por la creación de una entidad pública propietaria de suelo rural}

Los ejemplos señalados permiten constatar que el futuro de las Agroaldeas adolecen de unas bases débiles para oponerse con éxito a otros desarrollos de infraestructuras o sectores económicos más atrayentes para las administraciones locales.

Así mismo, queda patente la demanda de espacio para usos agrícolas, de forma que sirven tanto para paliar los efectos sobre las explotaciones que tiene el desarrollo de infraestructuras como para impulsar la nueva implantación de jóvenes agricultores que después de un periodo formativo desean integrarse en el sector primario. Si lo que se pretende es que las Agroaldeas sirvan para promocionar la agricultura, facilitar la incorporación de jóvenes al sector, fomentar la mejora técnica y de gestión de las explotaciones, etc., y que a su vez las inversiones realizadas en estas áreas sean viables económicamente y atractivas para los futuros agricultores, es fundamental plantearse el desarrollo y gestión de los Agroaldeas de forma diferente a la actual ${ }^{13}$.

12 Los Planeamientos que ordenan a una escala de actuación próxima a la urbanística, como los Planes Territoriales Parciales, adelantan un planteamiento con mayor incidencia en el medio rural. En el caso de San Sebastián, según el avance de este documento (2003), las grandes piezas del medio físico libres de asentamientos urbanos, pero cercanas a éstos y a las redes de comunicaciones son las principales reservas para las futuras posibles implantaciones dotacionales, configurándose como «vacíos de oportunidad». Señala que se debe evaluar cuidadosamente la ordenación de estas áreas con distintas alternativas, su preservación estricta o su especial regulación como zonas de interés agroganadero o forestal, hasta su ocupación urbanística intensiva, pasando por todo tipo de fórmulas intermedias. Lo que no debe hacerse es ignorar las potencialidades de desarrollo urbanístico intensivo que atesoran para el área funcional.

13 De hecho, el Plan de Jóvenes Agricultores de la Comunidad Autónoma del País Vasco (2004) plantea actuar en la promoción de las agroaldeas y del suelo agrario en general, como herramienta básica en su desarrollo. Las opciones planteadas pasan por crear una bolsa de suelo agrario disponible, gestionada por una entidad de intervención pública y dirigida a facilitar la creación de nuevas explotaciones o a mejorar las características de las ya existentes. La naturaleza del suelo es principalmente de dos tipos: suelo agrario o suelo procedente de la realización de infraestructuras. Las agroaldeas es uno de los campos a los que dan preferencia en su desarrollo. 
La necesidad de buscar un mejor sistema de organización nos lleva a volver la vista hacia el modelo de industrialdeas, gestionadas a través de Sociedades Públicas, en las que se implican las distintas Administraciones (Ayuntamientos, Diputaciones, Gobierno Vasco), con sociedades que ostentan la titularidad del suelo y la responsabilidad de su desarrollo.

Frente a los polígonos industriales la agroaldea se presenta como un modelo que adolece de multitud de debilidades. En este caso no hay una entidad de gestión superior, de ámbito comunitario y con capacidad para dinamizar una iniciativa que pueda intervenir en la captación de suelo para estos menesteres. La institución promotora, las Asociaciones de Desarrollo Rural, apenas tienen respaldo económico ni político, no son entidades públicas y dependen de la voluntad de las instituciones locales, con decisiones frecuentemente cambiantes. Se hace necesario, por tanto, reconducir un ejemplo adecuado como sistema de producción a las carencias de suelo del País Vasco Atlántico.

Un primer paso a seguir en la consecución de un modelo de gestión ha de partir necesariamente de una reflexión comarcalizada, que recoja una valoración del volumen de producción y del número aproximado de explotaciones a desarrollar, con una estimación de la superficie necesaria para instalar las mismas. Una vez localizadas las necesidades han de ser localizadas sobre el territorio y señalarlo en los documentos de ordenación correspondientes (PTP, PDR, PTS,... $)^{14}$. Sólo de este manera otras administraciones comenzarán a tomar en consideración la importancia económica y territorial de estas nuevas realidades espaciales ${ }^{15}$.

Otro paso necesario estriba en la propia orientación del modelo que pretendemos impulsar. En este sentido consideramos necesaria la creación por parte de los Departamentos de Agricultura de las distintas Administraciones de una Sociedad Pública, encargada de realizar los estudios de viabilidad necesarios, la adquisición del suelo, el desarrollo del mismo y la adjudicación-control de las parcelas resultantes, sin desprenderse de la propiedad de las mismas, de forma que el propietario interlocutor frente a cualquier Administración sea la propia Sociedad. Esto es, la experiencia de los polígonos industriales enseña que probablemente la única posibilidad de asegurar la continuidad y estabilidad permanente de un Agroaldea, viene de la existencia de un ente autónomo, con capacidad de acceder a la propiedad del suelo y que sea capaz de defender el suelo destinado para estas actividades, de forma que se pueda evitar que los agricultores queden desprotegidos.

De hecho, la toma de conciencia de la pérdida de suelo productivo que la agricultura va a sufrir como consecuencia de la programación de una gran infraestructura como es el tren de alta velocidad $^{16}$, lleva a plantearse por parte de las instituciones la creación de una entidad de suelo

14 En el marco de la planificación territorial actual todavía es la prospección municipal la que realiza la ordenación efectiva del territorio de la comarca. Sin embargo, a partir de la aprobación de las Directrices de Ordenación Territorial (DOT) en 1997, existen instrumentos de planificación que prevalecen sobre el planeamiento municipal y que eligen la comarca como ámbito de ordenación. Estos son los Planes Territoriales Parciales, la mayoría de ellos actualmente en proceso de aprobación.

15 Pocas son las experiencias en las que le medio rural haya sido estrictamente calificado en documentos de ordenación territorial más generales. Una de las escasas excepciones la constituye el Avance del Plan Sectorial Agroforestal y del Medio Natural del País Vasco, en el que se apuesta por una clasificación en función del carácter estratégico de la explotación agraria, concepto que, aunque tímidamente, comienza a ser desarrollado en documentos de planificación urbanística, entre otros en el Plan General de Donostia-San Sebastián (2004), en este momento en proceso de revisión y discusión.

16 Como señala GÓMEZ OREA (1992), la realización de infraestructuras en áreas de agricultura extensiva es una actividad compatible sometida a estudio de impacto ambiental, de ahí la justificación del trazado. Sin embargo, no lo es en aquellos casos en los que se trata de ecosistemas íntegros y paisajes escénicos, y el área afectada bien pudiera tener dicha calificación y aunque este aspecto apenas es planteado al menos obliga a preocuparse por sus implicaciones en este ámbito, un hecho que se produce en el planteamiento de la red ferroviaria vasca. 
rural que, aprovechando la envergadura de los movimientos de tierra que la obra a efectuar va a generar, compense con suelo, en la medida de sus posibilidades, a los agricultores afectados. La iniciativa es aprovechada por las instituciones de desarrollo agrario para proponer la creación de una entidad de gestión de suelo rural ${ }^{17}$ que aglutine otras posibilidades de gestión de tierras agrarias, además de las surgidas a partir de la realización del tren de alta velocidad.

Consecuentemente, los departamentos implicados, Transportes y Obras Públicas, y el de Agricultura y Pesca, representados por el Consejero de Transportes y el Consejero de Agricultura respectivamente, junto a los representantes de los Departamentos de Agricultura de las diferentes Diputaciones Forales organizan el proyecto de Decreto de creación de la Sociedad de Gestión del Banco de Suelo, por medio del cual la Comunidad Autónoma gestiona y administra los bienes inmuebles de naturaleza rústica afectados por la vía férrea del tren de velocidad alta en primer lugar. En el proyecto se contempla la gestión de diferentes formas de adquisición de terreno agrícola, entre otras: permutas, ceses anticipados en la actividad agraria, expropiaciones, ejercicio de los derechos de adquisición preferente... ${ }^{18}$; gestión de las fincas sobrantes y de propietarios desconocidos; la gestión de los sobrantes de terreno expropiado y de los lugares, preferentemente vaguadas, para deposito de materiales sobrantes de excavación; y gestión de los procesos de reversión que puedan derivarse de la ejecución de la obra de infraestructura ${ }^{19}$.

Las bases teóricas para crear una entidad de gestión de suelo agrario están, por tanto, ya diseñadas ${ }^{20}$. Es necesario, sin embargo, dar el paso necesario y poner en funcionamiento esta entidad que, además de los objetivos que persigue, ha de crear un apartado específico dirigido a la obtención de suelo y a la promoción de agroaldeas.

La función de esta entidad, además de retener suelo que pueda llegar mediante las diferentes vías que han sido analizadas, ha de ser la de conseguir que dicho soporte sea de su propiedad. No es suficiente con acceder a cesiones de suelo o derechos de uso. Es necesario retener o comprar la propiedad de determinadas parcelas que posteriormente van a ser utilizadas como agroaldeas ${ }^{21}$.

17 La demanda de un órgano público de intervención en el mercado de la tierra agraria es una solicitud ya tradicional de los sindicatos agrarios vascos. Así lo señala el Sindicato EHNE (2002), que apunta como punto de partida la constatación de la existencia de un problema, tierra, que en el ámbito a actual, la CAV, se define como la dificultad o imposibilidad de acceso por parte del productor agrario al factor básico de producción, la tierra, por no existir un mercado de tierra, sino de suelo, y no existir instrumentadas formas de acceso a ese factor de producción distintas al mercado.

18 De hecho, esta es la fórmula principal propuesta por los sindicatos agrarios que operan en la Comunidad Autónoma, la intermediación en operaciones de personas que quieran acogerse al régimen de ayudas por cese anticipado de la actividad agraria (EHNE, 1992), aunque dado el escaso resultado que la medida a tenido en las provincias más urbanas, Gipuzkoa y Bizkaia, la medida se nos antoja insuficiente.

19 El PTS Agroforestal y del Medio Natural propone gestionar un patrimonio compuesto por unos bienes inmuebles formados por el nuevo suelo generado con los depósitos de sobrantes de excavación generados por obras de infraestructura, fincas sobrantes y de propietarios desconocidos de la concentración parcelaria, suelo ocioso de las administraciones locales y fincas cedidas voluntariamente por sus propietarios para ser gestionadas por la entidad, especialmente de agricultores acogidos al plan de jubilaciones.

20 Se ha de reconocer, sin embargo, que el origen de la idea de crear bolsas o bancos de suelo hay que situarlo en el régimen sobre cese anticipado tras la reforma de la PAC de 1992. El reglamento CEE 2079/92 previó la creación de servicios y redes privadas pero con autorización pública para organizar las trasmisiones de tierras. Igualmente, se establecía que los estados miembros podían asimismo crear un organismo que se hiciera cargo de las tierras cedidas y se comprometiera a cederlas a personas que satisfagan las condiciones recogidas en el reglamento.

21 Si las propuestas realizadas van por esta línea, los pasos que hasta el momento se han dado están muy lejos de llegar al objetivo de crear una entidad de gestión de suelo rural. En este momento, según IKT (2004), tan sólo se ha avanzado en una propuesta de diseño de una Oficina de Intermediación para el suelo agrario afectado por el trazado de la infraestructura de velocidad alta «Y vasca» y que se centra en comunicar e informar al afectado del alcance y de los modelos de minimización del impacto de la infraestructura. Entre sus objetivos no está el de crear una entidad de gestión de suelo agrario más amplia y que tuviera la opción de acceder a la propiedad del suelo. 
Otro avance más en la organización del funcionamiento de las agroaldeas ha de venir de la necesaria reordenación de la función que actualmente cumplen las Asociaciones de Desarrollo Rural. En la medida en que estas entidades canalizan recursos económicos de Ayuntamientos y Diputaciones pueden contribuir en la ejecución de las mejoras que las distintas parcelas necesitan, tienen capacidad para dirigir las obras y proyectos a realizar, detectan oferta y demanda de suelo existente puesto que trabajan sobre el terreno y son las únicas entidades sectoriales con capacidad de llevar un control del funcionamiento de las agroaldeas, una vez que las parcelas hayan sido adjudicadas.

Por tanto, parece necesario instaurar en cada agroaldea la figura de la Asociación Mercantil que ya hemos desarrollado en el caso de los polígonos industriales, en la que tuvieran participación la entidad de gestión de suelo rural a crear para el conjunto de la Comunidad Autónoma Vasca, como propietaria de suelo, y las Asociaciones de Desarrollo Rural que correspondan en cada caso, en la medida en realizan mejoras económicas en las parcelas. De este modo, ante problemas originados como consecuencia de conflictos de usos entre actividades distintas, las entidades interesadas en adquirir estos suelos además de abonar los costos propios de la actividad que se desarrolla en las agroaldeas, aportarán el valor económico de un suelo que ya no es de su propiedad y que permitirá reubicar esas explotaciones en otro lugar, en principio próximo, puesto que será exigencia de la Asociación de Desarrollo Rural que tal recolocación se realice en la comarca.

Por lo demás, se considera correcto mantener la filosofía que caracteriza actualmente a los polígonos rurales, especialmente la apuesta por arrendamientos a largo plazo con precios simbólicos o asequibles, que permitan al nuevo productor entrar a competir en el mercado en condiciones similares a las de agricultores propietarios de suelo. Otro tanto podemos señalar de la gestión de los polígonos rurales que ha de ser llevada tal y como se desarrolla actualmente, es decir, de manera individualizada con la colaboración puntual de las Asociaciones de Desarrollo Rural. La diferencia principal se establece en que han de ser las entidades agrarias las que han de retener la propiedad del suelo, recabar las plusvalías que su posible recalifación pueda originar y garantizar, de ese modo, la continuidad de las explotaciones que hayan apostado por instalarse en las agroaldeas.

\section{Horticultura e industria. A modo de conclusión}

Con el ejemplo de la agroaldea de San Marcos se pone en evidencia la falta de suelo disponible en el periurbano de San Sebastián, que lleva a la agricultura a buscar espacio en terrenos límites, con claras dificultades de asentamiento, al que se le unen problemas de olores (es un vertedero activo) y de imagen para las explotaciones y el sector ${ }^{22}$.

La falta de suelos adecuados para el desarrollo de la actividad agrícola en el País Vasco es una realidad contrastada. La incorporación de nuevos agricultores al sector agroganadero en todo el País Vasco Atlántico es mínima debido, entre otras razones, a la imposibilidad de obtener o arrendar terrenos a un precio adecuado.

22 No son las únicas instalaciones ubicadas en superficies de estas características (recientemente se ha inaugurado un invernadero para planta forestal en una escombrera de una mina en Asturias) pero sí al menos, según la información que poseemos, las únicas instaladas sobre un vertedero de residuos sólidos urbanos. También se han llegado a recuperar escombreras para este tipo de usos. Concretamente, en 1995 se inician los trabajos de recuperación de una escombrera procedente de la mina Puramarabele, dentro de los planes de diversificación que la empresa HUNOSA está realizando en Asturias. En este caso estaba realizada con escombros procedentes de mina y lavado (ARQUITECTURA DEL PAISAJE, n 35, 1997, p. 22). La particularidad de la de San Marcos está en que se asienta sobre una base inestable, que tardará al menos 15 años en alcanzar una completa estabilidad (HAGINA, $\mathrm{n}^{\circ} 12,1997$, p. 41). 
Los destinatarios de estas parcelas generalmente son agricultores ya experimentados. El horticultor profesional cuenta con una ventaja añadida, ya que la incorporación a estos espacios requiere de una inversión cuantiosa y lo que es más importante, de un mercado propio ya consolidado. Mientras el proceso de expansión del suelo urbano y la creación de nuevas infraestructuras continúe, éste será el destinatario principal pues verá que su explotación se va reduciendo o que carece de posibilidades de ampliación. La incorporación de nuevos productores, aunque menor, también se está produciendo y la única vía real de acceso a la consecución de una explotación es precisamente accediendo a esta tipología de parcelas. De hecho, las previsiones de incorporación de jóvenes ajenos al sector se orientan hacia esta actividad preferentemente y tienen en la agroaldea uno de sus principales puntos de apoyo.

Esta iniciativa es un paliativo al problema de espacio en un área urbana, puesto que da opciones a horticultores necesitados de suelo. Surge, por tanto, como respuesta a la escasez de suelo a precios asequibles para el agricultor y su desarrollo viene potenciado por la inseguridad que provoca el estar sometido a una presión urbana intensa.

La idea también tiene un origen urbano, el polígono industrial de promoción pública, anterior a las agroaldeas y referente en la configuración de las mismas. De la industrialdea retoma algunas de sus concepciones básicas, preferentemente la pretensión de ser una actividad dirigida a la promoción de una actividad productiva, potenciada desde la iniciativa pública y fundamentada en la oferta de suelo. También toma de esta concepción la necesidad de que sea una entidad pública la que acondicione y urbanice las parcelas que, posteriormente, cada particular va a poner en funcionamiento.

La agroaldea, sin embargo, también aporta sus particularidades, propias de la necesidad de adecuación a las demandas de la actividad agraria. Entre éstas, cabe señalar la apuesta por la continuidad del suelo en manos públicas, utilizando arrendamientos a largo plazo y de una cuantía simbólica. Se pretende que el horticultor acceda al mercado en condiciones competitivas con horticultores ya instalados. Por otro lado, mediante una renta a largo plazo se garantiza que el agricultor pueda amortizar la inversión realizada, además de asegurar el uso al que originalmente iba dirigida la parcela.

El modelo desarrollado, sin embargo, adolece de graves problemas originados por la propia debilidad de su constitución y que han quedado en evidencia con el paso de los años. El hecho de que la entidad que promueve la agroaldea no sea la propietaria del suelo y que éste continúe en manos municipales impide, ante conflictos por su utilización, que puedan reubicarse las explotaciones en terrenos adquiridos a partir de las plusvalías originadas en la venta del suelo que utiliza. En estos casos, aún no materializados, con la agroaldea desaparecen las explotaciones instaladas en ella.

Por todo ello se hace necesario una renovación del sistema utilizado en la realización de las agroaldeas, que pasa por aprovechar la solidez y experiencia de su homónimo industrial. En este sentido, el contar con una entidad de gestión de suelo para usos agrario, que adquiriera en propiedad aquellos terrenos sobre los que se instalan las agroaldeas y que gestionara con las distintas Asociaciones de Desarrollo Rural el funcionamiento y la promoción de las mismas, es la herramienta que el desarrollo de esta modalidad requiere.

\section{Bibliografía}

ALBERDI COLLANTES, J.C. (1999): «Agroaldea: un concepto a desarrollar». Hagina, $\mathrm{n}^{\mathrm{o}} 20, \mathrm{pp} .42-44$.

ALBERDI COLLANTES, J.C. (2002): Horticultura y ocio en Gipuzkoa: nuevas formas de ocupación espacial. Nimbus $n^{\circ}$ 7-8. Pp. 21-37. 
ARDATZA (1988...): Publicación quincenal de la Unión de Ganaderos y Agricultores Vascos. Vitoria-Gasteiz.

ARQUITECTURA DEL PAISAJE (1997): «Recuperación de una escombrera minera para producción forestal». Arquitectura del Paisaje, $\mathrm{n}^{\circ} 35$, pp. 22-23.

COLMENAR, E. (1988): «La alegría de la huerta». MOPU. Revista del Ministerio de Obras Públicas y Urbanismo, $\mathrm{n}^{\circ} 10$, pp. 58-63.

GÓMEZ OREA, D. (1992): Planificación rural. Ministerio de Agricultura, Pesca y Alimentación. Editorial Agrícola Española, S.A. Madrid.

HAGINA (1993 ....): La revista Agrícola para la Cornisa Cantábrica. Oiartzun (Gipuzkoa).

IKT (2003): «Estudio del sector agrario en el Territorio histórico de Gipuzkoa». Diputación Foral de Gipuzkoa. Donostia. Inédito.

IKT (2003): «Oficina de Intermediación del Suelo Afectado por el trazado del la Y vasca». Gobierno Vasco. Inédito.

SANTAMARÍA, P. (2001): Los jóvenes en el sector agrario vasco (una revisión a partir de los censos agrarios). IKT (Vitoria). Informe Inédito.

TORRES ENJUTO, C. (1995): «Industria y Territorio en Bizkaia» Colección Tesis Doctorales. Instituto Vasco de la Administración Pública. Bilbao.

VERGARA, A. -Dir.- (1997): Directrices de Ordenación Territorial de la Comunidad Autónoma del País Vasco. 
